\section{Research Square}

\title{
ZNF674-AS1 suppresses non-small cell lung cancer growth through the miR-423-3p/p21 axis
}

\author{
Yu Liu \\ Wuzhou Medical University \\ Risheng Huang \\ Wenzhou Medical University \\ Deyao Xie \\ Wenzhou Medical University \\ Xiaoming Lin \\ Wenzhou Medical University \\ Liangcheng Zheng ( $\nabla$ dr_lczheng@163.com ) \\ Wenzhou Medical University https://orcid.org/0000-0002-8207-4198
}

Primary research

Keywords: cell cycle arrest, lung cancer, miRNA, ZNF674-AS1

Posted Date: October 1st, 2020

DOI: https://doi.org/10.21203/rs.3.rs-66110/v2

License: (c) (i) This work is licensed under a Creative Commons Attribution 4.0 International License.

Read Full License 


\section{Abstract}

Background: ZNF674-AS1, a recently characterized long noncoding RNA, shows prognostic significance in hepatocellualar carcinoma and glioma. However, the expression and function of ZNF674-AS1 in nonsmall cell lung cancer (NSCLC) is unclear.

Methods: In this work, we investigated the expression of ZNF674-AS1 in 83 pairs of NSCLC specimens and adjacent noncancerous lung tissues. The clinical significance of ZNF674-AS1 in NSCLC was analyzed. The role of ZNF674-AS1 in NSCLC growth and cell cycle progression was explored.

Results: Our data show that ZNF674-AS1 expression is decreased in NSCLC compared to normal tissues. ZNF674-AS1 downregulation is significantly correlated with advanced TNM stage and decreased overall survival of NSCLC patients. Overexpression of ZNF674-AS1 inhibits NSCLC cell proliferation, colony formation, and tumorigenesis, which is accompanied by a G0/G1 cell cycle arrest. Conversely, knockdown of ZNF674-AS1 enhances the proliferation and colony formation of NSCLC cells. Biochemically, ZNF674AS1 overexpression increases the expression of p21 through downregulation of miR-423-3p. Knockdown of p21 or overexpression of miR-423-3p blocks ZNF674-AS1-mediated growth suppression and G0/G1 cell cycle arrest. In addition, ZNF674-AS1 expression is negatively correlated with miR-423-3p in NSCLC specimens.

Conclusions: ZNF674-AS1 suppresses NSCLC growth by downregulating miR-423-3p and inducing p21. This work suggests the therapeutic potential of ZNF674-AS1 in the treatment of NSCLC.

\section{Background}

Lung cancer is the leading cause of cancer-related deaths among males worldwide $(1,2)$. Non-small cell lung cancer (NSCLC) accounts for over $80 \%$ of all lung cancer cases. Although advances have been made in therapeutic methods, the 5-year survival rate of patients with advanced NSCLC is less than $20 \%(3,4)$. Therefore, exploration of the molecular mechanisms governing NSCLC progression is important to develop effective therapies against this disease.

Long noncoding RNAs (IncRNAs) are a class of transcripts lacking protein-coding potential, with over 200 nucleotides in length (5). Accumulating evidence indicates that IncRNAs are aberrantly expressed in various cancer types and play a critical role in tumor progression $(6,7)$. For example, IncRNA JPX is upregulated in metastatic lung cancer and shows the capacity to promote lung cancer growth and metastasis (6). It has been suggested that IncRNAs regulate gene expression through interaction with microRNAs (miRNAs), which are small noncoding RNAs of 22-24 nucleotides in length $(8,9)$. Specially, IncRNAs may act as a sponge of miRNAs to release miRNA targets $(10,11)$. Also, IncRNAs can suppress the expression of miRNAs (12).

Although several IncRNAs such as JPX (6), UCA1 (7), and JHDM1D-AS1 (13) have been reported to coordinate lung cancer progression, most aberrantly expressed IncRNAs in lung cancer remain 
uncharacterized. The IncRNA ZNF674 antisense RNA 1 (ZNF674-AS1) is downregulated in hepatocellular carcinoma (HCC) (14). Moreover, ZNF674-AS1 downregulation is significantly correlated with distant metastasis, clinical stage, histopathologic grade, and poor prognosis in patients with HCC (14). Another study analyzing the Chinese Glioma Genome Atlas microarray dataset revealed that ZNF674-AS1 serves as an unfavorable prognostic factor for glioma (15). These preliminary results imply that ZNF674-AS1 may play a cell context-dependent role in tumor progression. However, the role of ZNF674-AS1 in NSCLC has not yet been clarified.

In the current study, we investigated the expression and clinical significance of ZNF674-AS1 in NSCLC. We performed gain- and loss-of-function experiments to determine the impact of ZNF674-AS1 on NSCLC aggressive phenotype. In addition, we identified the key miRNAs involved in the action of ZNF674-AS1 in NSCLC.

\section{Materials And Methods}

\section{Patients and tissues}

A total of 83 paired tumor samples and corresponding normal lung tissues were collected from NSCLC patients who received surgery at our hospital. Clinicopathological information of the patients is summarized in Supplementary Table S1. None was given any anticancer treatment before surgery. Tissue specimens were immediately frozen in liquid nitrogen and stored at $-80^{\circ} \mathrm{C}$.

\section{Cell culture}

NSCLC cell lines A549, H1299, H358, and PC9 were cultured in Dulbecco's Modified Eagle's Medium (DMEM) supplemented with $10 \%$ fetal bovine serum (FBS; Invitrogen, Carlsbad, CA, USA) at $37^{\circ} \mathrm{C}$ with $5 \%$ $\mathrm{CO}_{2}$ atmosphere. BEAS-2B cells were cultured in growth factor-supplemented medium (BEGM; Lonza, Walkersville, MD, USA). These cell lines were purchased from the Type Culture Collection of Chinese Academy of Science (Shanghai, China). No mycoplasma infection was detected in the cell lines used in this study.

\section{Quantitative real-time PCR (qRT-PCR) analysis}

Total RNA was extracted using TRIzol reagent (Invitrogen) and reverse-transcribed into cDNA using the Superscript III Reverse Transcriptase Kit (Invitrogen). qRT-PCR analysis of ZNF674-AS1 and p21 was performed using the following primers: ZNF674-AS1 forward, 5'-GCAGTGAATTACTGCTCATTC-3' and reverse, 5'-GCCACAGATCAGGTGCTTCT-3'; p21 forward, 5'-GCCCAGTGGACAGCGAGCAG-3' and reverse, 5'GCCGGCGTTTGGAGTGGTAGA-3' (16). Glyceraldehyde-3-phosphate dehydrogenase (GADPH) was used as an internal control. For quantification of mature miRNAs, total RNA was reverse-transcribed using the Taqman miRNA Reverse Transcription kit (Applied Biosystems, Carlsbad, CA, USA) and amplified using the TaqMan miRNA assay system (Applied Biosystems). U6 was used as an endogenous control. The relative gene expression was determined by the $2^{-\Delta \Delta C T}$ method (17). 


\section{Plasmids, small interfering RNAs (siRNAs), and miR-423-3p mimic}

A fragment containing ZNF674-AS1 was amplified by PCR and cloned to pcDNA3.1(+) expression vector. The p21-targeting siRNA (sip21) was purchased from Invitrogen. miR-423-3p mimic was purchased from Sigma-Aldrich. Two independent siRNAs targeting ZNF674-AS1 were synthesized by Sangon Biotechnology (Shanghai, China), with the target sequences listed as follows: ZNF674-AS1 siRNA\#1, 5'CCTAGATGGCTGTTGTTAT-3', and ZNF674-AS1 siRNA\#2, 5'-ATCTGATGTTAACAGTTGT-3'. Cell transfection was performed using Lipofactamine 3000 transfection reagent (Invitrogen), following the manufacturer's instruction.

\section{MTT assay}

Cells were plated in 96 -well plates $\left(5 \times 10^{3}\right.$ cells per well). After culturing for $24-72 \mathrm{~h}$, cells were collected and assessed by the 3-(4,5-dimethylthiazol-2-yl)-2,5-diphenyl-2H-tetrazolium bromide (MTT) assay. In brief, MTT $\left(0.5 \mathrm{mg} / \mathrm{ml}\right.$; Sigma-Aldrich, St. Louis, MO, USA) was added to each well and cultured at $37^{\circ} \mathrm{C}$ for $4 \mathrm{~h}$. The purple precipitates were dissolved in dimethyl sulfoxide (Sigma-Aldrich). Absorbance was measured at $490 \mathrm{~nm}$.

\section{Colony formation assay}

Colony formation assay was performed as described previously (18). Cells were seeded onto 6-well plates (600 cells per well). After incubation for 10 days, cells were fixed and stained with crystal violet (SigmaAldrich). The number of colonies per well was counted.

\section{Animal studies}

Male BALB/c nude mice, 5 weeks of age, were acclimated to the facility environment (a 12-h light/dark cycle, $23 \pm 2^{\circ} \mathrm{C}$, and $50 \%$ humidity) for 1 week. Xenograft tumors were generated by injecting stably transfected $\mathrm{A} 549$ cells $\left(2 \times 10^{6}\right)$ to the flanks of the mice. Tumor volume was measured every week. After 4 weeks mice were euthanatized, and tumors were weighed.

\section{Immunohistochemistry}

Xenograft tumors were fixed and sectioned. The sections were deparaffinized, probed with anti-Ki-67 antibody (Sigma-Aldrich) in a humidified chamber, and then incubated with horseradish peroxidase (HRP)-conjugated secondary antibody. Signals were developed using 3,3'-diaminobenzidine solution (Sigma-Aldrich). The sections were counterstained with hematoxylin.

\section{Cell cycle analysis}

For analysis of cell cycle progression, cells were fixed with $70 \%$ ethanol and stained with $50 \mu \mathrm{g} / \mathrm{mL}$ propidium iodide (PI) in the presence of $50 \mu \mathrm{g} / \mathrm{mL}$ RNAse A (Sigma-Aldrich). Stained cells were analyzed by flow cytometry. 


\section{Western blot analysis}

Cells were lysed in ice-cold RIPA lysis buffer supplemented with a protease inhibitor cocktail (SigmaAldrich). Protein samples ( $30 \mu \mathrm{g} / \mathrm{lane}$ ) were separated by sodium dodecyl sulfate polyacrylamide gel electrophoresis and transferred onto polyvinylidene fluoride membranes. The membranes were incubated overnight at $4^{\circ} \mathrm{C}$ with the primary antibodies recognizing p21 and GAPDH. These antibodies were purchased from Cell Signaling Technology (Danvers, MA, USA). The membranes were then incubated with secondary antibodies conjugated to HRP (Santa Cruz Biotechnology, Santa Cruz, CA, USA) for $1 \mathrm{~h}$. Enhanced chemiluminescent reagents (Millipore, Billerica, MA, USA) were used to visualize bound antibodies.

\section{Statistical analysis}

Data are expressed as mean \pm standard deviation. Significant differences were analyzed by the Student's $t$ test or one-way analysis of variance. The relationship of ZNF674-AS1 with clinicopathological parameters was analyzed using the Chi-square test. Survival analysis was performed by the Kaplan-Meier method. Pearson correlation analysis was done to determine the correlation between ZNF674-AS1 and miR-423-3p. $P<0.05$ was considered statistically significant.

\section{Results}

\section{ZNF674-AS1 is downregulated and predicts poor prognosis in NSCLC}

To explore the expression of ZNF674-AS1 in NSCLC, we analyzed the expression of ZNF674-AS1 in 83 pairs of NSCLC specimens and adjacent noncancerous lung tissues. The results showed that ZNF674AS1 expression was significantly decreased in NSCLC relative to normal tissues $(P=0.0069$; Figure $1 \mathrm{~A})$. According to the median level of ZNF674-AS1 in tumor specimens, the NSCLC patients were divided into 2 groups: high vs. low ZNF674-AS1 groups. Downregulation of ZNF674-AS1 was significantly correlated with advanced TNM stage ( $P=0.0010$; Figure 1B). The Kaplan-Meier analysis further indicated that NSCLC patients with low ZNF674-AS1 levels had a shorter overall survival than those with high ZNF674AS1 levels $(P<0.0001 ;$ Figure 1C). In addition, analysis of ZNF674-AS1 expression in 504 lung adenocarcinoma specimens using KM Plotter (http://kmplot.com/analysis/) validated that low ZNF674AS1 expression was associated with decreased overall survival (Figure 1D). These data suggest that ZNF674-AS1 downregulation may contribute to NSCLC progression.

\section{ZNF674-AS1 suppresses NSCLC cell proliferation and colony formation}

Consistent with the clinical findings, ZNF674-AS1 expression was downregulated in the NSCLC cell lines tested compared to BEAS-2B bronchial epithelial cells (Figure 2A). To determine the role of ZNF674-AS1 in NSCLC cell growth and invasion, ZNF674-AS1 was ectopically overexpressed in both A549 and H1299 cells (Figure 2B), which had low levels of endogenous ZNF674-AS1. The growth of NSCLC cells was significantly suppressed by overexpression of ZNF674-AS1 (Figure 2C). Moreover, ZNF674-AS1-mediated 
growth suppression was confirmed in colony formation assays (Figure 2D). However, ZNF674-AS1 overexpression had no impact on the invasion ability of NSCLC cells (Supplementary Figure S1).

We further investigated the effect of ZNF674-AS1 on A549 cell tumorigenesis in a nude mouse model. Tumor volume measurements demonstrated that ZNF674-AS1-overexpressing A549 xenografts were significantly smaller than control xenografts (Figure 3A). At 4 weeks after cell inoculation, tumor weight in the ZNF674-AS1-overexpressing group was $\sim 4$-fold lower than that in the control group (Figures $3 B$ and 3C). Histological analysis confirmed that there were fewer ki-67-positive proliferating cells in ZNF674AS1-overexpressing xenografts than in control xenografts (Figure 3D). Collectively, these data indicate that ZNF674-AS1 can exert a growth-suppressing activity in NSCLC.

\section{Knockdown of ZNF674-AS1 accelerates NSCLC cell growth}

To further gain insight into the role of ZNF674-AS1 in NSCLC, we performed ZNF674-AS1 knockdown experiments in $\mathrm{H} 358$ cells using siRNA technology. As measured by qPCR analysis, transfection with ZNF674-AS1 siRNA led to a reduction of ZNF674-AS1 abundance in H358 cells by $60-70 \%$ (Figure 4A). The downregulation of ZNF674-AS1 increased the proliferation of H358 cells, as revealed by the MTT assay (Figure 4B). Moreover, colony formation was enhanced in ZNF674-AS1-depleted H358 cells (Figure 4C). Hence, ZNF674-AS1 exerts anti-proliferative effects on NSCLC cells.

\section{ZNF674-AS1 causes a G0/G1 cell cycle arrest through upregulation of p21}

Next, we asked whether ZNF674-AS1-mediated growth suppression was ascribed to induction of cell cycle arrest. Flow cytometric analysis of cell cycle distribution revealed that overexpression of ZNF674AS1 increased the number of cells in the $\mathrm{G} 1$ phase and decreased the number of cells in the $S$ phase (Figure 5A), indicating a cell cycle arrest at the G0/G1 phase. We further examined the expression of a number of cell cycle-related genes. The results demonstrated that overexpression of ZNF674-AS1 led to a marked increase of p21 protein in both A549 and H1299 cells (Figure 5B). Other cell cycle genes tested (cyclin D1 and p27) remained unaffected (data not shown). Moreover, the level of p21 mRNA was elevated in ZNF674-AS1-overexpressing NSCLC cells (Figure 5C). Therefore, we speculated that p21 was involved in the growth-suppressing activity of ZNF674-AS1. To validate the hypothesis, we performed p21 knockdown experiments (Figure 5D). As shown in Figures 5E and 5F, p21 knockdown rescued ZNF674AS1-mediated growth suppression and G0/G1 cell cycle arrest. These results indicate that ZNF674-AS1 inhibits NSCLC cell growth via upregulation of p21.

\section{ZNF674-AS1 antagonizes miR-423-3p to derepress p21}

Due to the interplay between IncRNAs and miRNAs in the regulation of gene expression $(6,7)$, we asked whether ZNF674-AS1 could promote p21 through interaction with specific miRNAs. To address this issue, we examined the effect of ZNF674-AS1 overexpression on the expression of multiple candidate miRNAs (19-25), which have shown the ability to repress p21. Interestingly, overexpression of ZNF674-AS1 caused a decline in the level of miR-423-3p but not miR-224, miR-33b-3p, miR-208a, miR-639, miR-572, or miR-663 
(Figure 6A). Overexpression of miR-423-3p attenuated ZNF674-AS1-dependent induction of p21 (Figure 6B). Moreover, ZNF674-AS1-mediated growth suppression and cell cycle arrest was reversed by miR-423$3 p$ overexpression (Figures $6 \mathrm{C}$ and $6 \mathrm{D}$ ). In addition, there was an inverse correlation between the expression of ZNF674-AS1 and miR-423-3p in NSCLC specimens ( $r=-0.407, P=0.0002$; Figure 6E).

\section{Discussion}

Previous studies have identified a number of IncRNAs that are dysregulated in NSCLC and serve as potential prognostic biomarkers for this malignancy $(13,26)$. For instance, upregulation of IncRNA SNHG3 by E2F1 is associated with a low overall survival rate of patients with NSCLC (26). In this study, we report a novel prognostic factor for NSCLC. We show that ZNF674-AS1 is downregulated in NSCLC relative to corresponding normal lung tissues. The downregulation of ZNF674-AS1 is associated with advanced TNM stage and reduced overall survival of NSCLC patients. The potential of ZNF674-AS1 as a prognostic biomarker has also been described in HCC (14) and glioma (15).

Biologically, ZNF674-AS1 overexpression inhibits the proliferation and tumorigenesis of NSCLC cells. In contrast, the invasive capacity of NSCLC cells is not affected by ZNF674-AS1 overexpression. Our data provide first evidence for the role of ZNF674-AS1 in modulating tumor progression. The tumorsuppressive activity of ZNF674-AS1 may provide an explanation for the clinical relationship between ZNF674-AS1 downregulation and reduced overall survival of NSCLC patients. Multiple IncRNAs have exhibited the capacity to regulate cell cycle progression $(27,28)$. For instance, knockdown of IncRNA EPIC1 leads to growth suppression and G0/G1 cell cycle arrest in pancreatic cancer cells (27). The IncRNA EPIC1 has been shown to promote cell cycle progression in cancer cells through interaction with MYC (28). Our data indicate ZNF674-AS1 as a negative regulator of cell cycle progression in NSCLC cells. Specially, ectopic expression of ZNF674-AS1 arrests NSCLC cells at the G0/G1 phase. Therefore, ZNF674AS1 inhibits NSCLC growth, at least partially through inducing G0/G1 cell cycle arrest.

Mechanistically, ZNF674-AS1 selectively stimulates the expression of p21 in NSCLC cells. p21 belongs to the Cip and Kip family of CDK inhibitors (29). It exerts biological activities primarily by inhibiting the kinase activity of CDKs $(30,31)$. Induction of p21 has been shown to impair the G1/S cell cycle transition in NSCLC cells by inhibiting the activation of CDK2 complexes (30). Interestingly, we show that knockdown of p21 prevents growth suppression of NSCLC cells induced by ZNF674-AS1. Therefore, ZNF674-AS1-mediated antiproliferative activity in NSCLC cells depends on the upregulation of p21.

It has been suggested that IncRNAs can regulate gene expression through interaction with miRNAs $(6,7)$. For instance, IncRNA XIST is capable of inducing p21 through interaction with miR-106b-5p in renal cell carcinoma (32). Similarly, Nrf2-IncRNA can promote the expression of p21 by sponging miR-128 and miR224 (33). Here, we show that ZNF674-AS1 overexpression suppresses the expression of miR-423-3p in NSCLC cells. miR-423-3p has been shown to promote lung cancer proliferation and invasion (34). Previous studies have reported p21 as a direct target of miR-423-3p $(23,35)$. Overexpression of miR-423$3 p$ was found to inhibit p21 expression in HCC (23) and colorectal cancer (35). Consistently, our data 
indicate that restoration of miR-423-3p blocks the induction of $\mathrm{p} 21$ by ZNF674-AS1 in NSCLC cells. Biologically, ZNF674-AS1-mediated anticancer activity is counteracted by miR-423-3p overexpression. Clinically, ZNF674-AS1 expression is negatively correlated with miR-423-3p in NSCLC tissues. Taken together, we suggest that ZNF674-AS1 exerts its suppressive activity against NSCLC through inhibition of miR-423-3p and subsequent derepression of p21 (Figure 6F).

\section{Conclusion}

We identify ZNF674-AS1 as a new growth suppressor in NSCLC. Induction of p21 due to miR-423-3p downregulation is responsible for ZNF674-AS1-mediated growth suppression. Our data suggest that restoration of ZNF674-AS1 represents a potential therapeutic strategy to treat NSCLC.

\section{Abbreviations}

IncRNA: long noncoding RNA; miRNA: microRNA; NSCLC: non-small cell lung cancer; Pl: propidium iodide; qRT-PCR: quantitative real-time PCR

\section{Declarations}

\section{Ethics statement}

Written informed consent for research was obtained from each patient. This study was approved by the Human Ethics Committee of the First Affiliated Hospital of Wenzhou Medical University (Wenzhou, China). All animal experiments were approved by the Animal Care and Use Committee of Wenzhou Medical University and performed in accordance with the Guide for the Care and Use of Laboratory Animals of Wenzhou Medical University.

\section{Author contributions}

YL and LCZ participated in study conception and data interpretation. $Y L, R S H, D Y X$, and $X M L$ performed experiments. YL and LCZ prepared the manuscript. All authors read and accepted the final version.

\section{Conflict of interest}

The authors declare that there is no potential conflict of interest.

\section{Funding}

This work was supported by the Natural Science Foundation of Zhejiang Province of China (LY17H160048).

\section{Consent for publication}

Not applicable 
Availability of data and materials

Not applicable

\section{Acknowledgements}

Not applicable

\section{References}

1. Siegel RL, Miller KD, Jemal A. Cancer statistics, 2019. CA Cancer J Clin. 2019;69:7-34.

2. Ferlay J, Colombet M, Soerjomataram I, Mathers C, Parkin DM, Piñeros M, Znaor A, Bray F. Estimating the global cancer incidence and mortality in 2018: GLOBOCAN sources and methods. Int J Cancer. 2019;144:1941-53.

3. Ouyang W, Yu J, Huang Z, Chen G, Liu Y, Liao Z, Zeng W, Zhang J, Xie C. Risk factors of acquired T790M mutation in patients with epidermal growth factor receptor-mutated advanced non-small cell lung cancer. J Cancer. 2020;11:2060-7.

4. Millares L, Barreiro E, Cortes R, Martinez-Romero A, Balcells C, Cascante M, Enguita AB, Alvarez C, Rami-Porta R, Sánchez de Cos J, Seijo L, Monsó E; Grupo Colaborativo en Cáncer de Pulmón CIBERES- CIBERONC- SEPAR - Plataforma Biobanco Pulmonar. Tumor-associated metabolic and inflammatory responses in early stage non-small cell lung cancer: Local patterns and prognostic significance. Lung Cancer. 2018;122:124-30.

5. Ren X, Chen C, Luo Y, Liu M, Li Y, Zheng S, Ye H, Fu Z, Li M, Li Z, Chen R. IncRNA-PLACT1 sustains activation of NF-KB pathway through a positive feedback loop with IKBa/E2F1 axis in pancreatic cancer. Mol Cancer. 2020;19:35.

6. Pan J, Fang S, Tian H, Zhou C, Zhao X, Tian H, He J, Shen W, Meng X, Jin X, Gong Z. IncRNA JPX/miR-33a-5p/Twist1 axis regulates tumorigenesis and metastasis of lung cancer by activating Wnt/ $\beta$-catenin signaling. Mol Cancer. 2020;19:9.

7. Chen X, Wang Z, Tong F, Dong X, Wu G, Zhang R. IncRNA UCA1 Promotes Gefitinib Resistance as a ceRNA to Target FOSL2 by Sponging miR-143 in Non-small Cell Lung Cancer. Mol Ther Nucleic Acids. 2019;19:643-53.

8. Chakraborty M, Hu S, Visness E, Del Giudice M, De Martino A, Bosia C, Sharp PA, Garg S. MicroRNAs organize intrinsic variation into stem cell states. Proc Natl Acad Sci U S A. 2020;117:6942-50.

9. Fehlmann T, Kahraman M, Ludwig N, Backes C, Galata V, Keller V, Geffers L, Mercaldo N, Hornung D, Weis T, Kayvanpour E, Abu-Halima M, Deuschle C, Schulte C, Suenkel U, von Thaler AK, Maetzler W, Herr C, Fähndrich S, Vogelmeier C, Guimaraes P, Hecksteden A, Meyer T, Metzger F, Diener C, Deutscher S, Abdul-Khaliq H, Stehle I, Haeusler S, Meiser A, Groesdonk HV, Volk T, Lenhof HP, Katus H, Balling R, Meder B, Kruger R, Huwer H, Bals R, Meese E, Keller A. Evaluating the Use of Circulating MicroRNA Profiles for Lung Cancer Detection in Symptomatic Patients. JAMA Oncol. 2020;6:1-10. 
10. Wang YG, Wang T, Shi M, Zhai B. Long noncoding RNA EPB41L4A-AS2 inhibits hepatocellular carcinoma development by sponging miR-301a-5p and targeting FOXL1. J Exp Clin Cancer Res. 2019;38:153

11. Zhao X, Su L, He X, Zhao B, Miao J. Long noncoding RNA CA7-4 promotes autophagy and apoptosis via sponging MIR877-3P and MIR5680 in high glucose-induced vascular endothelial cells. Autophagy. 2020;16:70-85.

12. Wan $Y$, Yang ZQ. LncRNA NEAT1 affects inflammatory response by targeting miR-129-5p and regulating Notch signaling pathway in epilepsy. Cell Cycle. 2020:1-13.

13. Yao G, Chen K, Qin Y, Niu Y, Zhang X, Xu S, Zhang C, Feng M, Wang K. Long Non-coding RNA JHDM1D-AS1 Interacts with DHX15 Protein to Enhance Non-Small-Cell Lung Cancer Growth and Metastasis. Mol Ther Nucleic Acids. 2019;18:831-40.

14. Zhang L, He T, Yan Y, Zhang Y, Zhou X, Huang P, Kong Y, Xie M, Zhang L, Sun Q, Zhou D, Xie H, Zhou L, Zheng S, Wang W. Expression and Clinical Significance of the Novel Long Noncoding RNA ZNF674-AS1 in Human Hepatocellular Carcinoma. Biomed Res Int. 2016;2016:3608914.

15. Luan F, Chen W, Chen M, Yan J, Chen H, Yu H, Liu T, Mo L. An autophagy-related long non-coding RNA signature for glioma. FEBS Open Bio. 2019;9:653-67.

16. Wang C, Chen Z, Ge Q, Hu J, Li F, Hu J, Xu H, Ye Z, Li LC. Up-regulation of p21(WAF1/CIP1) by miRNAs and its implications in bladder cancer cells. FEBS Lett. 2014;588:4654-64.

17. Livak KJ, Schmittgen TD. Analysis of relative gene expression data using real-time quantitative PCR and the 2(-Delta Delta C(T)) Method. Methods. 2001;25:402-8.

18. Huang RS, Zheng YL, Zhao J, Chun X. microRNA-381 suppresses the growth and increases cisplatin sensitivity in non-small cell lung cancer cells through inhibition of nuclear factor-KB signaling. Biomed Pharmacother. 2018;98:538-44.

19. Wang H, Zhu LJ, Yang YC, Wang ZX, Wang R. MiR-224 promotes the chemoresistance of human lung adenocarcinoma cells to cisplatin via regulating $\mathrm{G} 1 / \mathrm{S}$ transition and apoptosis by targeting p21(WAF1/CIP1). Br J Cancer. 2014;111:339-54.

20. Xu S, Huang H, Chen YN, Deng YT, Zhang B, Xiong XD, Yuan Y, Zhu Y, Huang H, Xie L, Liu X. DNA damage responsive miR-33b-3p promoted lung cancer cells survival and cisplatin resistance by targeting p21WAF1/CIP1. Cell Cycle. 2016;15:2920-30.

21. Tang Y, Cui Y, Li Z, Jiao Z, Zhang Y, He Y, Chen G, Zhou Q, Wang W, Zhou X, Luo J, Zhang S. Radiation-induced miR-208a increases the proliferation and radioresistance by targeting p21 in human lung cancer cells. J Exp Clin Cancer Res. 2016;35:7.

22. Lei ST, Shen F, Chen JW, Feng JH, Cai WS, Shen L, Hu ZW, Xu B. MiR-639 promoted cell proliferation and cell cycle in human thyroid cancer by suppressing CDKN1A expression. Biomed Pharmacother. 2016;84:1834-40.

23. Lin J, Huang S, Wu S, Ding J, Zhao Y, Liang L, Tian Q, Zha R, Zhan R, He X. MicroRNA-423 promotes cell growth and regulates $\mathrm{G}(1) / \mathrm{S}$ transition by targeting $\mathrm{p} 21 \mathrm{Cip} 1 /$ Waf1 in hepatocellular carcinoma. Carcinogenesis. 2011;32:1641-7. 
24. Zhang X, Liu J, Zang D, Wu S, Liu A, Zhu J, Wu G, Li J, Jiang L. Upregulation of miR-572 transcriptionally suppresses SOCS1 and p21 and contributes to human ovarian cancer progression. Oncotarget. 2015;6:15180-93.

25. Yi C, Wang Q, Wang L, Huang Y, Li L, Liu L, Zhou X, Xie G, Kang T, Wang H, Zeng M, Ma J, Zeng Y, Yun JP. MiR-663, a microRNA targeting p21(WAF1/CIP1), promotes the proliferation and tumorigenesis of nasopharyngeal carcinoma. Oncogene. 2012;31:4421-33.

26. Shi J, Li J, Yang S, Hu X, Chen J, Feng J, Shi T, He Y, Mei Z, He W, Xie J, Li S, Jie Z, Tu S. LncRNA SNHG3 is activated by E2F1 and promotes proliferation and migration of non-small-cell lung cancer cells through activating TGF- $\beta$ pathway and IL-6/JAK2/STAT3 pathway. J Cell Physiol. 2020;235:2891-900.

27. Xia P, Liu P, Fu Q, Liu C, Luo Q, Zhang X, Cheng L, Qin T, Zhang H. Long noncoding RNA EPIC1 interacts with YAP1 to regulate the cell cycle and promote the growth of pancreatic cancer cells. Biochem Biophys Res Commun. 2020;522:978-85.

28. Wang Z, Yang B, Zhang M, Guo W, Wu Z, Wang Y, Jia L, Li S; Cancer Genome Atlas Research Network, Xie W, Yang D. IncRNA Epigenetic Landscape Analysis Identifies EPIC1 as an Oncogenic IncRNA that Interacts with MYC and Promotes Cell-Cycle Progression in Cancer. Cancer Cell. 2018;33:706-20.

29. Abbas T, Dutta A. p21 in cancer: intricate networks and multiple activities. Nat Rev Cancer. 2009;9:400-14.

30. Bae KM, Wang H, Jiang G, Chen MG, Lu L, Xiao L. Protein kinase $C$ epsilon is overexpressed in primary human non-small cell lung cancers and functionally required for proliferation of non-small cell lung cancer cells in a p21/Cip1-dependent manner. Cancer Res. 2007;67:6053-63.

31. Vilgelm AE, Saleh N, Shattuck-Brandt R, Riemenschneider K, Slesur L, Chen SC, Johnson CA, Yang J, Blevins A, Yan C, Johnson DB, Al-Rohil RN, Halilovic E, Kauffmann RM, Kelley M, Ayers GD, Richmond A. MDM2 antagonists overcome intrinsic resistance to CDK4/6 inhibition by inducing p21. Sci Transl Med. 2019 Aug 14;11(505). pii: eaav7171. doi: 10.1126/scitransImed.aav7171.

32. Sun K, Jia Z, Duan R, Yan Z, Jin Z, Yan L, Li Q, Yang J. Long non-coding RNA XIST regulates miR106b-5p/P21 axis to suppress tumor progression in renal cell carcinoma. Biochem Biophys Res Commun. 2019;510:416-20.

33. Joo MS, Shin SB, Kim EJ, Koo JH, Yim H, Kim SG. Nrf2-IncRNA controls cell fate by modulating p53dependent Nrf2 activation as an miRNA sponge for PIk2 and p21 cip1. FASEB J. 2019;33:7953-69.

34. Wang R, Li G, Zhuang G, Sun S, Song Z. Overexpression of microRNA-423-3p indicates poor prognosis and promotes cell proliferation, migration, and invasion of lung cancer. Diagn Pathol. 2019;14:53.

35. Li HT, Zhang H, Chen Y, Liu XF, Qian J. MiR-423-3p enhances cell growth through inhibition of p21Cip1/Waf1 in colorectal cancer. Cell Physiol Biochem. 2015;37:1044-54.

\section{Figures}



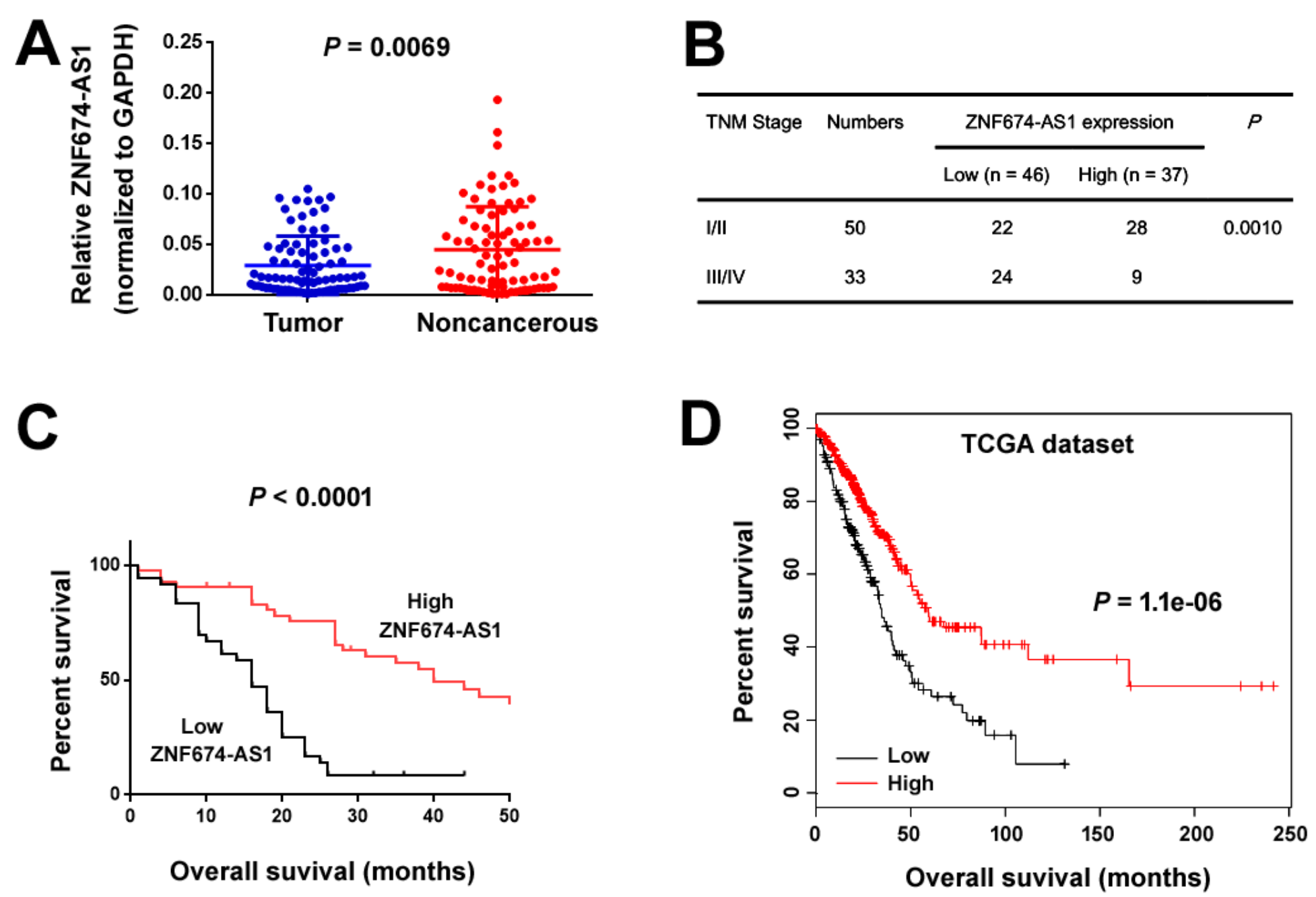

Figure 1

ZNF674-AS1 is downregulated and predicts poor prognosis in NSCLC. (A) Analysis of ZNF674-AS1 levels in 83 pairs of NSCLC specimens and adjacent noncancerous lung tissues. (B) Downregulation of ZNF674-AS1 was significantly associated with advanced TNM stage of NSCLC. (C) Kaplan-Meier analysis showed that reduced ZNF674-AS1 expression was associated with a shorter overall survival of NSCLC patients. (D) Based on the lung adenocarcinoma TCGA dataset included in KM Plotter, ZNF674AS1 expression was associated with decreased overall survival. 

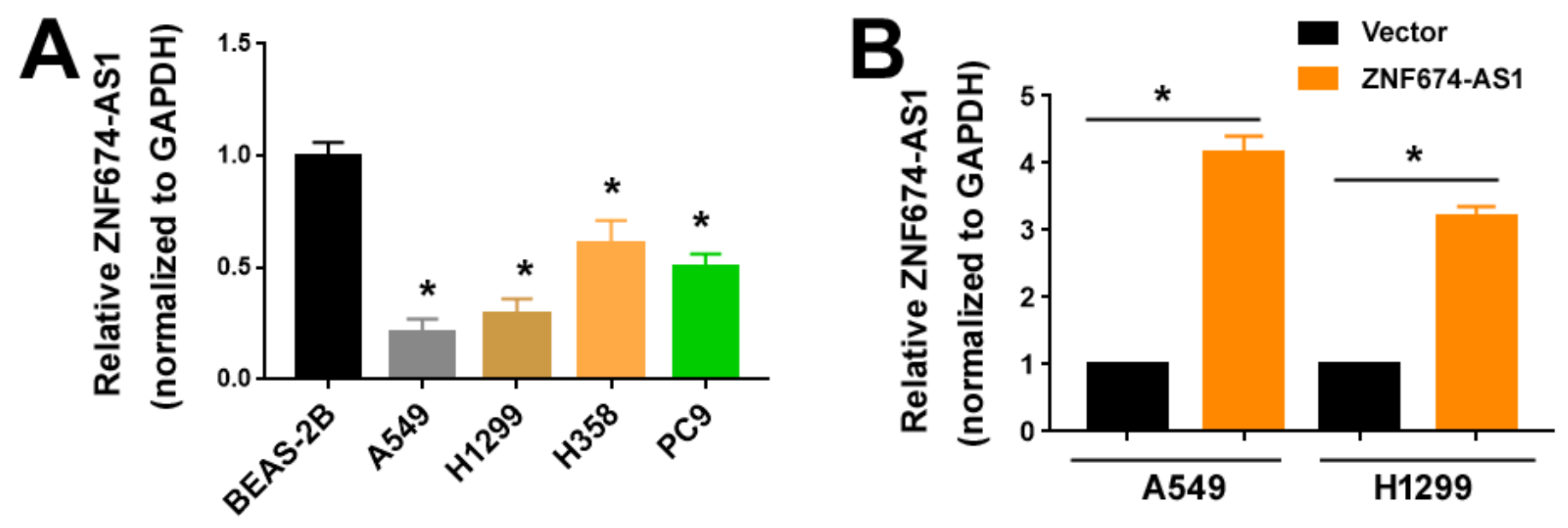

c
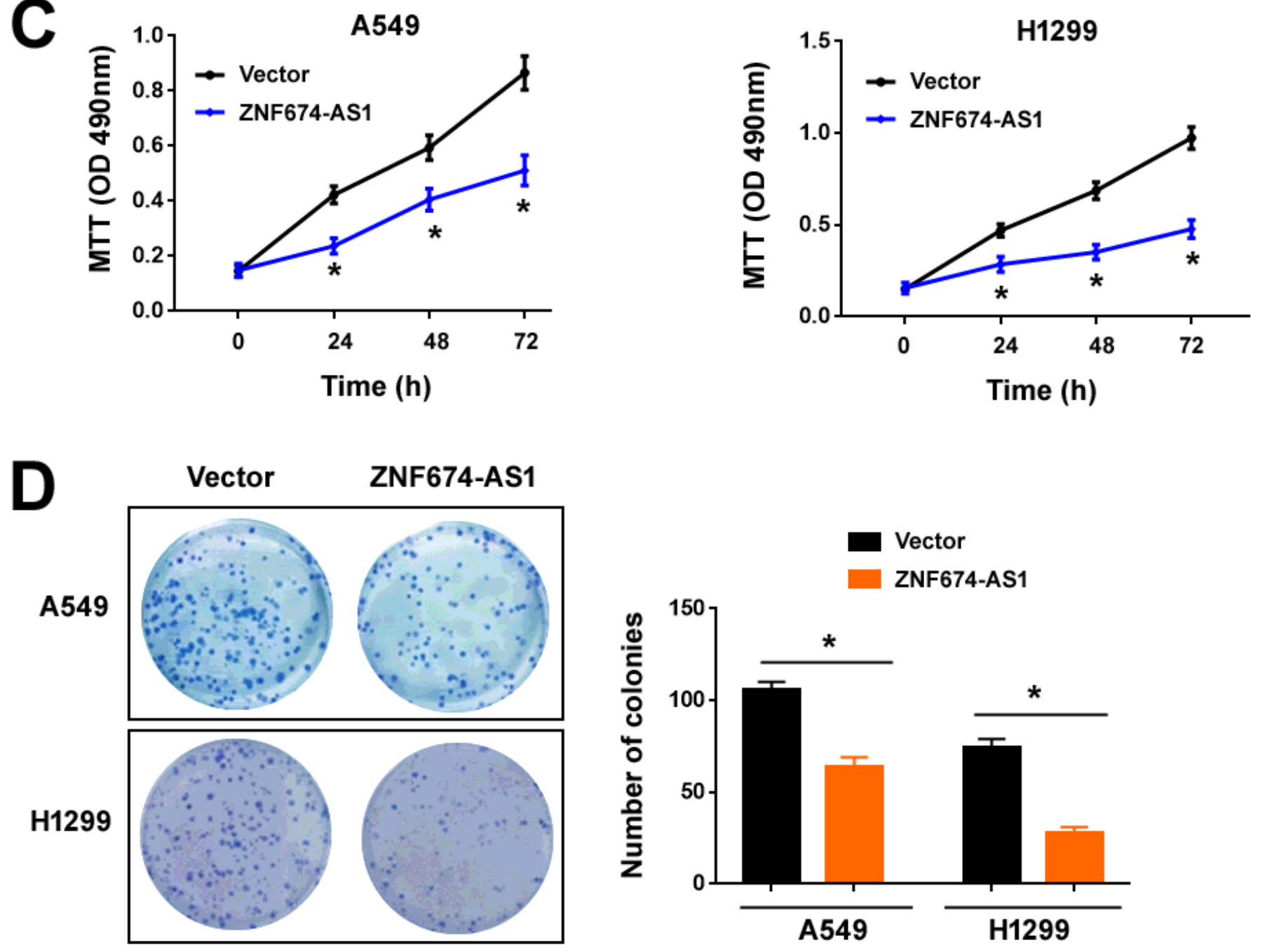

Figure 2

ZNF674-AS1 suppresses NSCLC cell proliferation and colony formation. (A) Expression of ZNF674-AS1 in indicated cell lines. ${ }^{*} \mathrm{P}<0.05$ compared to BEAS-2B cells. (B) Overexpression of ZNF674-AS1 in both A549 and H1299 cells. (C) As determined by MTT assay, NSCLC cell growth was suppressed by overexpression of ZNF674-AS1. (D) Colony formation assay showed that ZNF674-AS1 suppressed colony formation capacity of NSCLC cells. ${ }^{*} \mathrm{P}<0.05$ vs. Vector. 

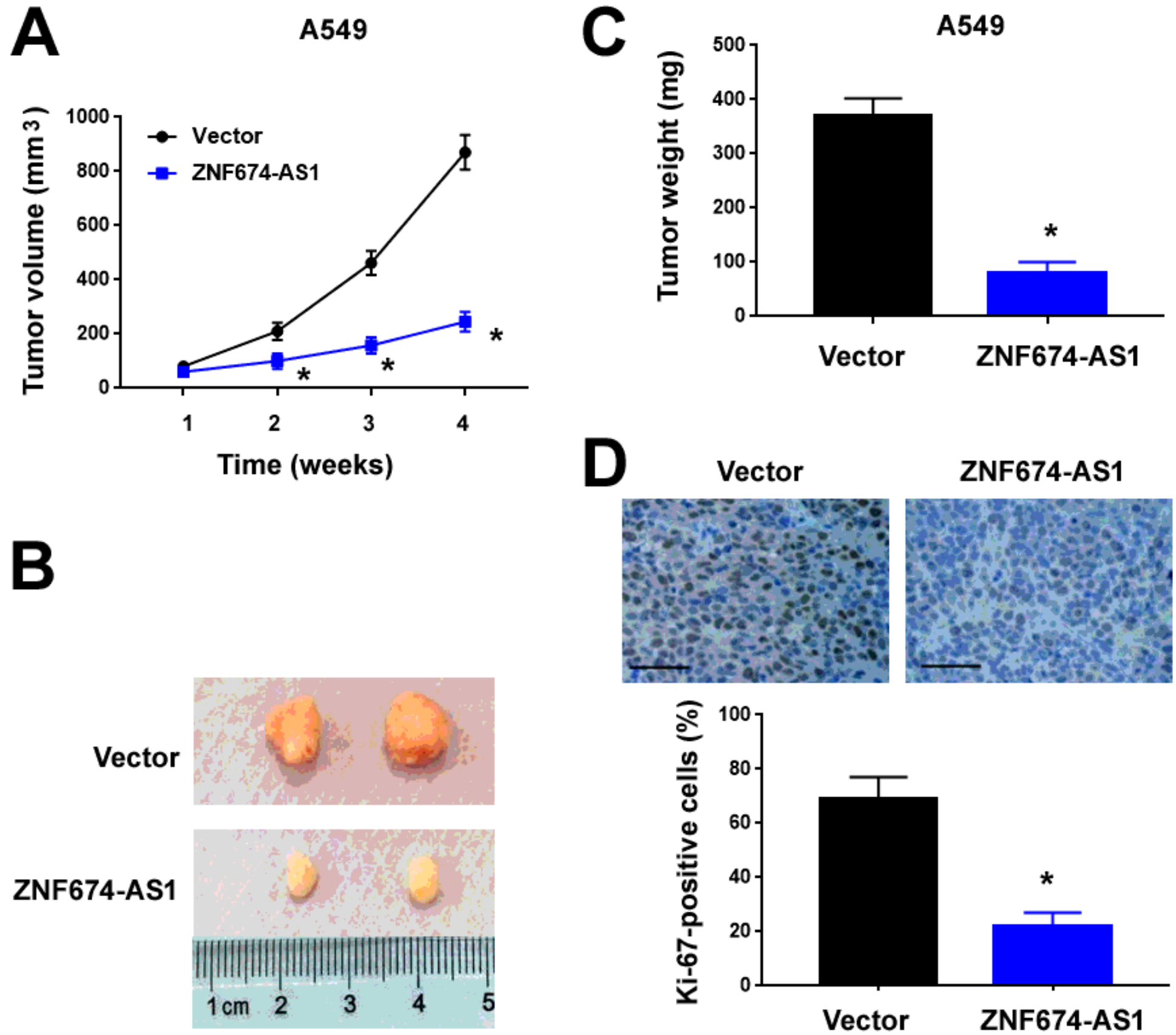

Figure 3

ZNF674-AS1 blocks tumorigenesis of A549 cells in vivo. (A) Assessment of the growth of xenograft tumors formed by ZNF674-AS1-overexpressing and control A549 cells. (B) Photographs of 2 representative xenograft tumors from each group. (C) Tumor weight was measured 4 weeks after cell inoculation. (D) Ki-67 immunostaining performed in xenograft tumors. Scale bar $=100 \mu \mathrm{m}$. Quantitative results of Ki-67 staining are shown in bottom panel. * $\mathrm{P}<0.05$ vs. Vector. 

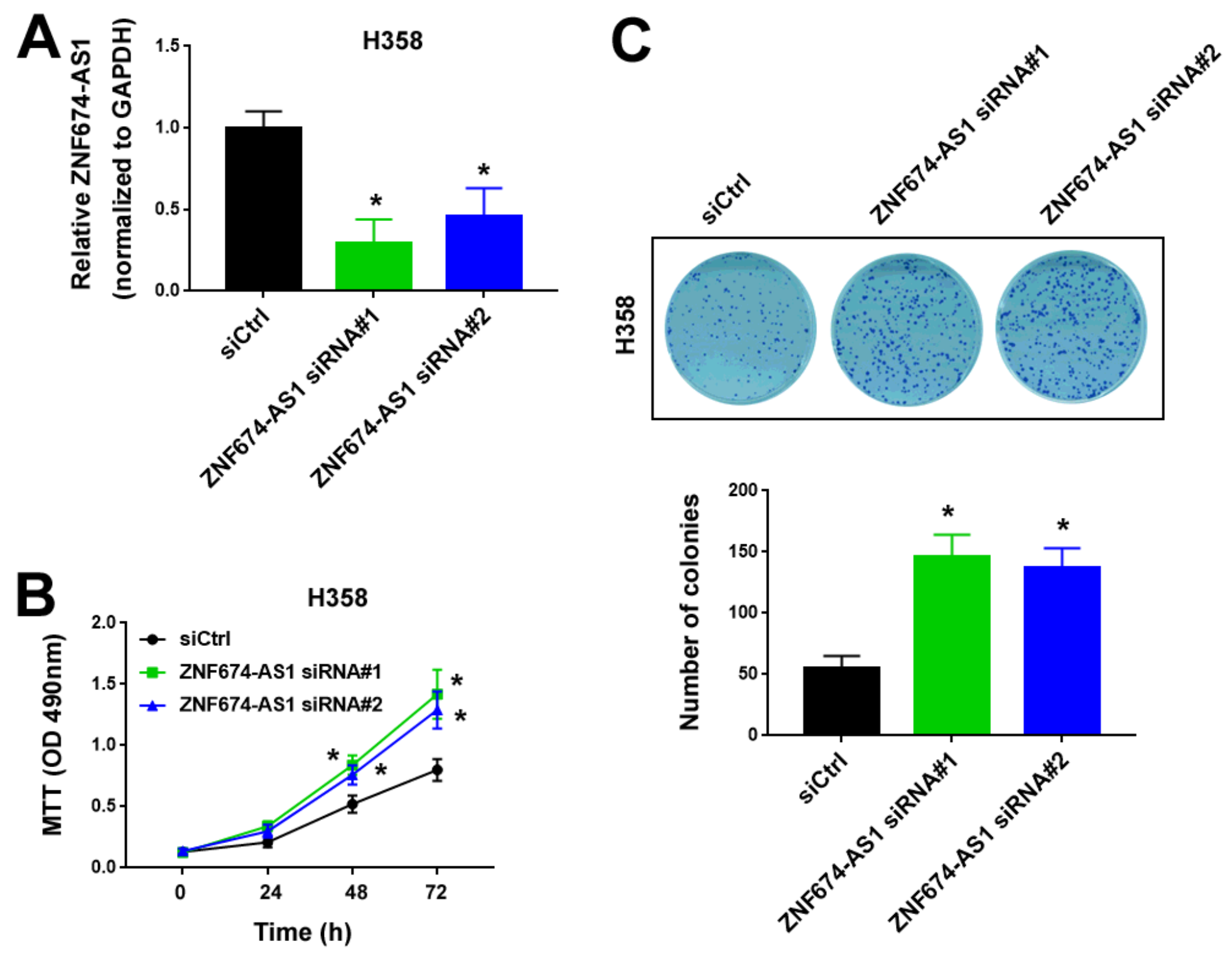

Figure 4

Knockdown of ZNF674-AS1 accelerates NSCLC cell growth and colony formation. (A) The level of ZNF674-AS1 transcript was analyzed in H358 cells transfected with control siRNA (siCtrl) or ZNF674-AS1targeting siRNAs. (B) MTT assay showed the induction of proliferation in ZNF674-AS1-depleted H358 cells. (C) Assessment of colony formation in $\mathrm{H} 358$ cells transfected with indicated siRNAs. ${ }^{*} \mathrm{P}<0.05$ vs. siCtrl. 
A
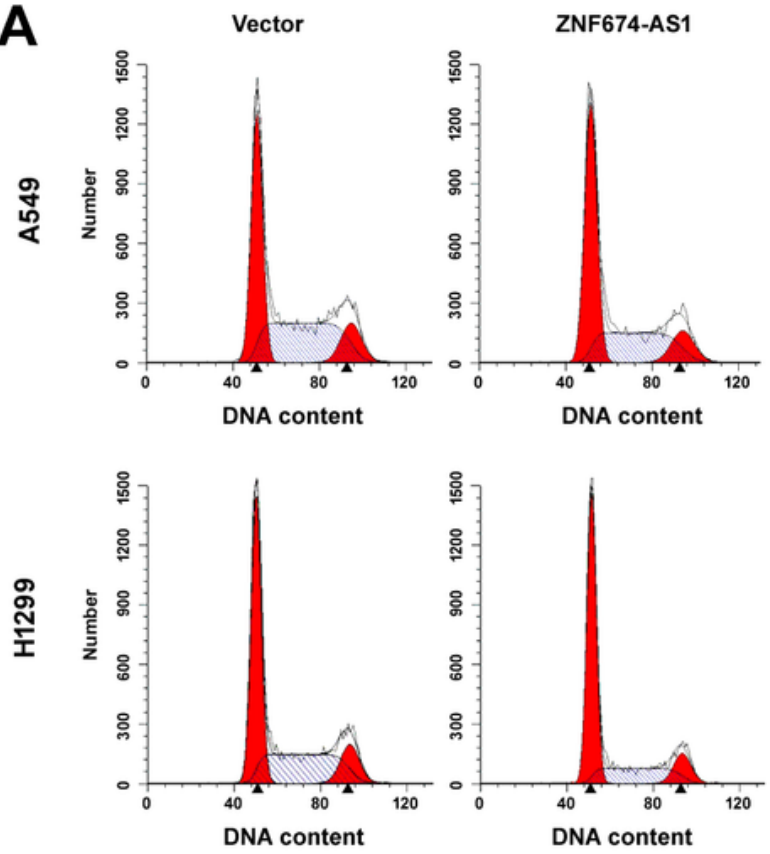

G2/M

s

- G0/G1
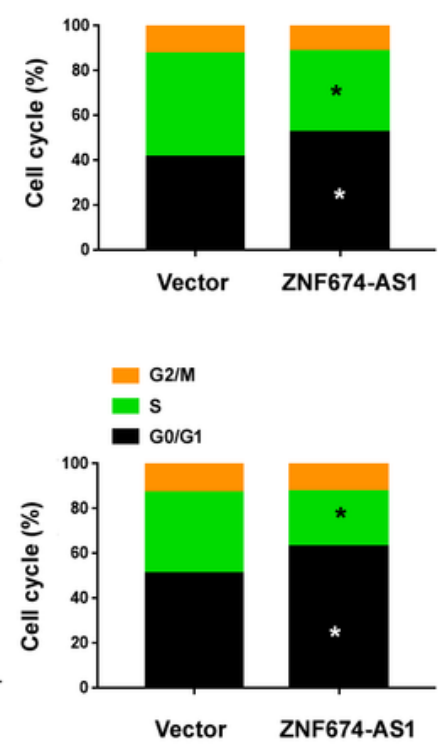

B

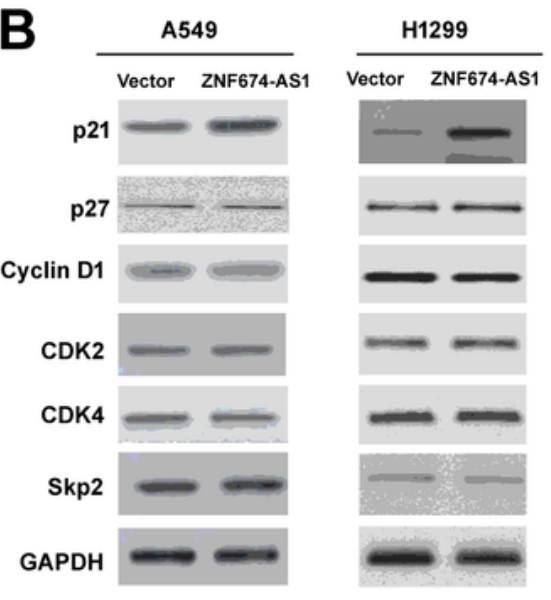

C

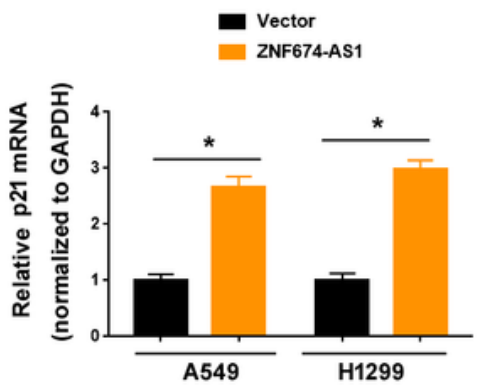

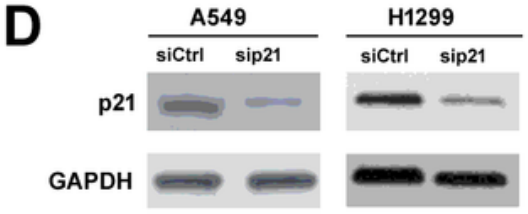

$\mathbf{E}$

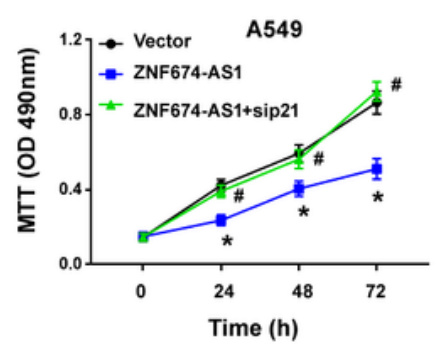

$\mathbf{F}$

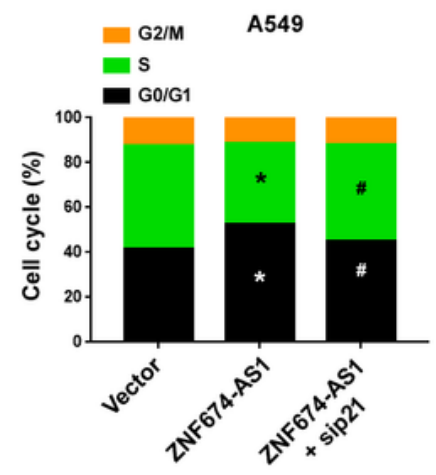

\section{Figure 5}

ZNF674-AS1 causes a G0/G1 cell cycle arrest through upregulation of p21. (A) Flow cytometric analysis showed that overexpression of ZNF674-AS1 arrested NSCLC cells at the G0/G1 phase. ${ }^{*} \mathrm{P}<0.05$ compared to vector-transfected cells. (B) Western blot analysis of indicated proteins. (C) ZNF674-AS1 overexpression increased the levels of p21 mRNA in NSCLC cells. ${ }^{*} \mathrm{P}<0.05$. (D) Knockdown of p21 in NSCLC cells by transfection of siRNAs. siCtrl: control siRNA; sip21: p21-targeting siRNA. (E) MTT assay 
showed that p21 knockdown rescued ZNF674-AS1-mediated growth of A549 cells. (F) Cell cycle analysis done in A549 cells transfected with indicated constructs. ${ }^{*} P<0.05$ compared to vector-transfected cells; \#P $<0.05$ compared to cells transfected with ZNF674-AS1 alone.
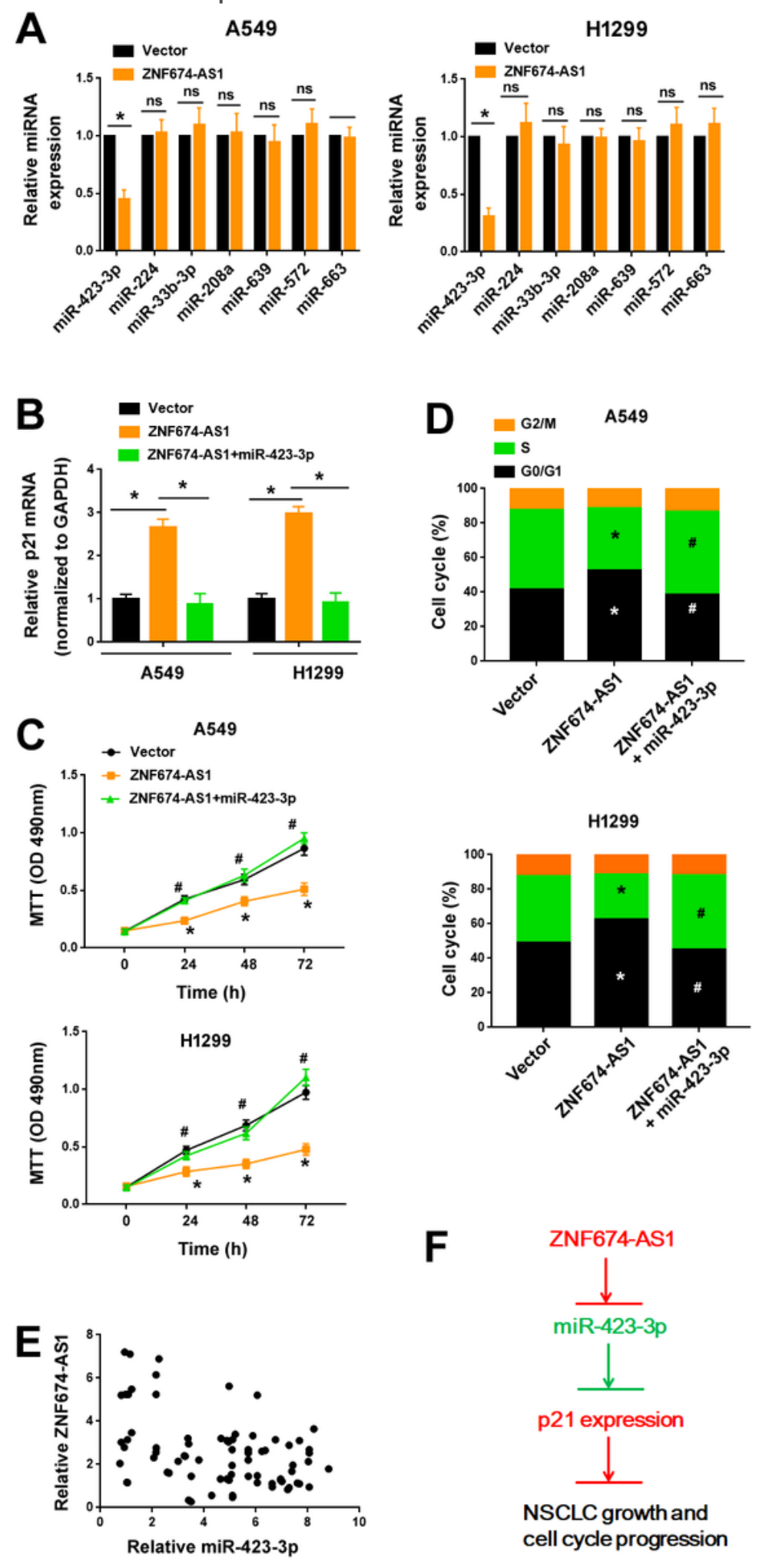

Figure 6

ZNF674-AS1 antagonizes miR-423-3p to derepress p21. (A) Analysis of candidate miRNAs in NSCLC cells transfected with vector or ZNF674-AS1. *P< 0.05. ns: no significance. (B) Analysis of p21 mRNA levels 
after transfection with indicated constructs. ${ }^{*} P<0.05$. (C) MTT assay showed that ZNF674-AS1mediated growth suppression was reversed by miR-423-3p. (D) Cell cycle analysis done in NSCLC cells transfected with indicated constructs. ${ }^{*} \mathrm{P}<0.05$ compared to vector-transfected cells; $\# \mathrm{P}<0.05$ compared to cells transfected with ZNF674-AS1 alone. (E) An inverse correlation was detected between the expression of ZNF674-AS1 and miR-423-3p in NSCLC specimens $(n=83)$. (F) Schematic model of the ZNF674-AS1/miR-423-3p/p21 pathway.

\section{Supplementary Files}

This is a list of supplementary files associated with this preprint. Click to download.

- Supplementarydata.pdf 